\title{
1 Whale shark rhodopsin adapted to its vertically wide-ranging \\ 2 lifestyle
}

4 Kazuaki Yamaguchi ${ }^{1, \#}$, Mitsumasa Koyanagi ${ }^{2, \#}$, Keiichi Sato ${ }^{3,4}$, Akihisa Terakita ${ }^{2, *}$,

5 Shigehiro Kuraku1,5,6*

$7 \quad{ }^{1}$ Laboratory for Phyloinformatics, RIKEN Center for Biosystems Dynamics

8 Research (BDR), Kobe, Japan.

92 Department of Biology and Geosciences, Graduate School of Science, Osaka City

10 University, Osaka, Japan.

$11{ }^{3}$ Okinawa Churashima Research Center, Okinawa Churashima Foundation,

12 Okinawa, Japan.

$13 \quad{ }^{4}$ Okinawa Churaumi Aquarium, Okinawa, Japan.

$14{ }^{5}$ Molecular Life History Laboratory, Department of Genomics and Evolutionary

15 Biology, National Institute of Genetics, Mishima, Japan.

$16{ }^{6}$ Department of Genetics, Sokendai (Graduate University for Advanced Studies),

17 Mishima, Japan.

$18 \quad$ \#equally contributed

19 *To whom correspondence may be addressed. Shigehiro Kuraku and Akihisa

20 Terakita

21 Email: skuraku@nig.ac.jp, terakita@osaka-cu.ac.jp

22 Keywords: visual opsin, rhodopsin, whale shark, spectral tuning, night blindness,

23 thermal stability 


\section{Abstract}

26 Spectral tuning of visual pigments often facilitates adaptation to new environments,

27 and it is intriguing to study the visual ecology of pelagic sharks with expanded

28 habitats. The whale shark, which dives into the deep sea of nearly 2,000 meters

29 besides near-surface filter-feeding, was previously shown to possesses the 'blue-

30 shifted' rhodopsin (RHO). In this study, our spectroscopy of recombinant whale

31 shark RHO mutants revealed the dominant effect of the novel spectral tuning

32 amino acid site 94 , which is implicated in congenital stationary night blindness of

33 humans, accounting for the blue shift. Thermal decay profiling revealed the

34 reduction of the thermal stability of whale shark $\mathrm{RHO}$, as typically observed for

35 cone opsins, which was experimentally shown to be achieved by the site 178 , as

36 well as 94 . The results suggest that these two sites cooperatively enhance the

37 visual capacity in both the deep sea and the sea surface, enabling exceptionally

38 wide vertical migration of this species. 
41 The whale shark Rhincodon typus, known as the largest extant fish, dives into the

42 deep sea of 2,000 meters while it forages with filter-feeding near the surface

43 (Tyminski et al., 2015). Because of its exceptionally wide vertical habitat range, it is

44 of great interest to investigate its visual ecology. Recent genome sequencing

45 confirmed degenerated visual opsin gene repertoires of this species, containing

46 only rhodopsin (RHO or Rh1) and long wavelength-sensitive opsin (LWS) (Hara et

47 al., 2018). Our previous spectroscopic analysis on the whale shark RHO pigment,

48 performed in vitro, uncovered a remarkable shift of the wavelength of the maximum

49 absorbance $(\lambda \max )$ to $478 \mathrm{~nm}$, from the presumable ancestral condition of $500 \mathrm{~nm}$

50 (Figure 1; Hara et al., 2018). As the light of approximately $480 \mathrm{~nm}$ is the least

51 attenuated in the deep sea, this finding suggested the reliance of the whale shark

52 on vision in the deep sea with the tuned $\mathrm{RHO}$, but the mechanism that allows this

53 so-called 'blue shift' has remained unknown. Interestingly, an in silico study on the

54 whale shark $\mathrm{RHO}$ has failed to predict candidate amino acid residues that account

55 for the blue shift (Fasick et al., 2019).

\section{Results}

58 We first focused on the zebra shark Stegostoma tigrinum (formerly, called S.

59 fasciatum), one of the phylogenetically closest extant species to the whale shark

60 (Figure 1; Naylor et al., 2012). The absorption spectrum of the zebra shark RHO

61 peaked at $500 \mathrm{~nm}$, as in the other close relative dwelling in shallow waters, the 
62 brownbanded bamboo shark (Figure 1; Hara et al., 2018). This substantiated the

63 uniqueness of the blue shift to the whale shark lineage.

Next, we scrutinized the sequence alignment of RHO orthologs of these

65 sharks and their relatives. This analysis did not reveal any amino acid substitution

66 unique to the whale shark among the previously characterized 'spectral tuning

67 sites' of the RHO, such as the sites 83, 292, and 299 (Hart et al., 2020; Musilova et

68 al., 2019; Yamaguchi et al., 2021; Figure 1-figure supplement 1). Among the

69 amino acid residues positioned within the range of $4.5 \AA$ from the chromophore 11-

70 cis-retinal (Palczewski et al., 2000), we identified two residues 94 and 178

71 substituted exclusively in the whale shark RHO (Ala94 and Phe178; Figure 1-

72 figure supplement 1). We examined the effect of each of these substitutions with

73 site-directed mutagenesis. Spectroscopic analysis of recombinant RHO mutants

74 revealed that the $\lambda$ max of whale shark RHO A94T and F178Y mutants shifted

75 toward longer wavelengths by 19 and $3 \mathrm{~nm}$, respectively, compared with that of the

76 wild-type (Figure 2A). Conversely, mutants with substitutions at these sites (T94A

77 and $\mathrm{Y} 178 \mathrm{~F}$ ) of zebra shark recapitulated the blue shift by 19 and $3 \mathrm{~nm}$ (Figure 2B).

78 This result shows a dominant effect of Ala94 in the blue shift of the whale shark

$79 \mathrm{RHO}$ and accounts for the $22 \mathrm{~nm}$ difference from the $\lambda$ max of zebra shark RHO by

80 cumulative effect of these two mutations. At the site 94 of $\mathrm{RHO}$, no case of natural

81 substitution has been reported as responsible for spectral tuning, except for the

82 T94I causing a certain type of human diseases including congenital stationary night

83 blindness (CSNB) (Al-Jandal et al., 1999; reviewed in Park, 2014). The molecular

84 basis of CSNB with T94I is explained by the substitution from a hydrophilic to 
85 hydrophobic residue, which leads to lower thermal stability via thermal

86 isomerization of the retinal and hydrolysis of the Schiff base (Singhal et al., 2016).

This observation in humans prompted us to investigate thermal stability of

88 the whale shark RHO (Figure 2C, D). Similar to the case of T94I included above

89 (Singhal et al., 2016), the A94I mutant of the whale shark RHO rapidly decayed at

$9037^{\circ} \mathrm{C}$, indicating that the site 94 also affects the thermal stability in the whale shark

91 RHO (Table 1 and Figure 2C). In fact, the wild-type whale shark RHO (Ala94) also

92 showed faster decay with a half-life $\left(\mathrm{t}_{1 / 2}\right)$ of 99.0 min than its A94T mutant $\left(\mathrm{t}_{1 / 2}=\right.$

$93173.3 \mathrm{~min})$ (Figure 2C-figure supplement 2, Table 1), indicating a decrease of

94 thermal stability by the T94A substitution. Interestingly, the whale shark RHO A94T

95 mutant has higher thermal stability than the wild-type but still lower than the zebra

96 shark RHO wild-type (Thr94, $\left.\mathrm{t}_{1 / 2}=346.6 \mathrm{~min}\right)$. This result is consistent with the

97 observation that the zebra shark RHO T94A mutant has higher thermal stability $\left(\mathrm{t}_{1 / 2}\right.$

$98=231.0 \mathrm{~min}$ ) than the whale shark RHO wild-type (Ala94, $\left.\mathrm{t}_{1 / 2}=99.0 \mathrm{~min}\right)$ (Figure

$99 \quad 2 \mathrm{C}, \mathrm{D})$.

We also scrutinized the site 178 , the other site substituted exclusively in the

101 whale shark RHO. The F178Y mutant of the whale shark RHO exhibited

102 comparable thermal stability $\left(t_{1 / 2}=231.0 \mathrm{~min}\right)$ to the A94T mutant, and introducing

103 both A94T and F178Y substitutions into the whale shark RHO recovered high

104 thermal stability $\left(t_{1 / 2}=346.6 \mathrm{~min}\right)$ close to that of the zebra shark wild-type (Figure

$1052 \mathrm{C}$ ). A parallel effect was observed in the zebra shark RHO; mutations at both sites

106 (T94A/Y178F) resulted in a severe decrease of thermal stability than either

107 mutation (Figure 2D). Taken together, substitutions at the two sites and their 
108 cumulative effect account for the lower thermal stability of the whale shark RHO

109 (Table 1), as in the blue shift. The rate of half-life difference between the wild-type

110 and the double mutant for the whale shark $(\approx 3.5$ times $)$ exceeded that for the

111 zebra shark $(\approx 2.5$ times) (Table 1$)$, which could be explained by possible influence

112 from other amino acid substitutions that accumulated in either evolutionary lineage.

114 Discussion

115 Blue shift of RHO resulting from deep-sea life has repeatedly been documented,

116 but mostly by studies on deep-sea 'specialists' (Hart et al., 2020; Musilova et al.,

117 2019). In contrast, the whale shark is reported to spend a considerable proportion

118 of the daytime on filter-feeding near the surface, besides occasional deep dives

119 down to nearly 2,000 meters (Tyminski et al., 2015). Although the whale shark is

120 capable of stabilizing deep body temperatures (Nakamura et al., 2020), the retina

121 is thought to be affected by wide-ranging water temperatures that depend on the

122 depth (e.g., 4.2 to $33.0^{\circ} \mathrm{C}$ reported in Tyminski et al., 2015). Our present study

123 revealed novel spectral tuning sites for blue shift of $\mathrm{RHO}$, Ala94 and Phe178,

124 which could have enabled its unique lifestyle with exceptionally wide vertical

125 migration.

126 Our study revealed a commonality of the substitution at the site 94 in the

127 blue-shifted spectrum and lowered thermal stability between the whale shark RHO

128 and the human RHO with a CSNB-causing mutation (T94I). In general, high

129 thermal stability is a critical feature of $\mathrm{RHO}$ to achieve a high signal-to-noise ratio

130 by reducing dark noise, which inherently enables dim-light vision (Yanagawa et al., 
131 2015). This functional nature of RHO constraining dark noise usually does not

132 permit the sites 94 or 178 to contribute to its spectral tuning. The co-existence of

133 these substitutions has not been observed in any visual opsins orthologous to $\mathrm{RHO}$

134 by our exhaustive search in the public sequence database (Supplementary Tables

1351 and 2).

In our experiment at $37^{\circ} \mathrm{C}$, the thermal stability of whale shark RHO

137 excelled that of the T94I mutant (Figure 2C and Table 1-figure supplement 2) but

138 is significantly lower than that of zebra shark (Figure $2 \mathrm{C}, \mathrm{D}$ ). The reduced thermal

139 stability of the whale shark $\mathrm{RHO}$ is reminiscent of cone opsins that have lower

140 thermal stability than RHOs to enable daylight vision. In fact, compared to the

141 human RHO (Janz et al., 2003), the whale shark RHO has lower thermal stability,

142 which is rather closer to that of mammalian LWS/MWS (Alexander et al., 2017;

143 Srinivasan et al., 2017) and chicken RH2 (Sato et al., 2018). Near the sea surface,

144 the cone opsin-like low thermal stability of whale shark $\mathrm{RHO}$ in high-temperature

145 water (Figure 2C) can facilitate the photoreceptor light adaptation suitable for the

146 bright light condition. While the whale shark has evolutionarily lost all cone opsins

147 except LWS (Hara et al., 2018), the whale shark RHO may compensate the

148 degenerative cone opsin repertoires by facilitating light adaptation. On the other

149 hand, in the deep sea, the functionality of whale shark RHO to reduce dark noise

150 would be maintained at low temperatures, as shown for $\mathrm{RHO}$ mutants with

151 persistent functionality at low temperatures (Janz et al., 2003). This speculation is 
152 compatible with the blue shift of the whale shark $\mathrm{RHO}$ that is thought to facilitate

153 dim-light sensing in the deep sea.

154 Our in vitro study that is supported by cross-species, genome-scale

155 sequence informatics provides the first case of spectral tuning of RHO by natural

156 substitutions at the sites 94 and 178 . The former plays a major role in the blue shift

157 analyzed in this study, while the pivotal role of the latter is regulating thermal

158 stability. The route of "rhodopsin tuning" we revealed is distinct from typical

159 solutions for deep-sea specialists and may have facilitated the habitat expansion of

160 this iconic species with vertically wide movement utilizing both deep sea and sea

161 surface.

163 Materials and Methods

164 cDNA sequencing. The eyes of a female zebra shark that was born from captive

165 breeding were sampled at the Okinawa Churaumi Aquarium in accordance with the

166 official guideline for animal experiments at Okinawa Churaumi Aquarium. Total

167 RNAs were extracted from these tissues using TRIzol Reagent (Life Technologies)

168 and used as a template in reverse transcription with the SMART cDNA Library

169 Construction Kit (Clontech). cDNA fragments amplified using the degenerate

170 primers designed based on the ortholog sequences of close relatives were

171 sequenced with a 3730xI DNA Analyzer (Thermo Fisher Scientific). The nucleotide

172 sequence of the zebra shark RHO is deposited in GenBank under the accession ID

173 MT625929. Our experiment was conducted in accordance with the institutional 
174 guideline Regulations for the Animal Experiments and approved by the Institutional

175 Animal Care and Use Committee (IACUC) of RIKEN Kobe Branch.

Rhodopsin expression and purification. The RHO expression vectors designed

178 as described previously (Koyanagi et al., 2015) based on the abovementioned

179 zebra shark sequence as well as the previously reported whale shark sequence

180 (Accession ID MT625928; Hara et al., 2018) were synthesized and cloned by a

181 custom service at GenScript. The RHO mutants were constructed using the KOD-

182 Plus-Mutagenesis Kit (Toyobo). The vectors were transfected into HEK293S cells

183 by the calcium-phosphate method. The cells were cultured for 48 hours for protein

184 expression. The RHO pigments were reconstituted as described previously (Hara

185 et al., 2018). An excessive amount of 11-cis-retinal was added to the cells

186 recovered by centrifugation, and the pigments were constituted by overnight

187 incubation at $4{ }^{\circ} \mathrm{C}$ in the dark. The cells were lysed in $1 \%(w / v) n$-dodecyl- $\beta$-D-

188 maltoside (DM) in 50 mM HEPES buffer ( $\mathrm{pH}$ 6.5) containing $140 \mathrm{mM} \mathrm{NaCl}$ (Buffer

$189 \mathrm{P})$, and the pigments were solubilized. The pigments were bound to 1D4-agarose, 190 washed with $0.02 \%(w / v)$ DM in Buffer $\mathrm{P}$, and eluted with $0.02 \%(\mathrm{w} / \mathrm{v}) \mathrm{DM}$ in Buffer $191 \quad \mathrm{P}$ containing the 1D4 peptide.

193 Spectroscopic measurements. Absorption spectra of purified pigments were 194 measured with the V-750 UV-VIS Spectrophotometer (JASCO International).

195 Yellow light was supplied by the light source with Y-50 glass cutoff filter (Toshiba).

196 The maximum absorption spectra were measured at $4{ }^{\circ} \mathrm{C}$ in the dark. Thermal 
197 decay assay of RHO was performed with incubation at $37^{\circ} \mathrm{C}$ in the dark, and the

$198 \lambda$ max was measured every two minutes. At the end of the measurement, the

199 pigments were irradiated with light, and the spectra of the bleached pigments were

200 measured.

201

202 In silico scan of RHO sequences. The NCBI nr database as of June 2020 was

203 downloaded for a BLASTP search using the whale shark RHO sequence as a

204 query. The collected peptide sequences were aligned with the program MAFFT

205 v7.299b using the L-INS-i method (Katoh \& Standley, 2013). The aligned

206 sequences were trimmed with trimAl v1.4.rev15 using the '-automated1' option,

207 followed by the removal of gapped sites using the '-nogaps' option (Capella-

208 Gutiérrez et al., 2009). The maximum-likelihood tree was inferred with RAxML

209 v8.2.8 using the PROTCATWAG model. For evaluating the confidence of nodes,

210 the rapid bootstrap resampling with 1,000 replicates was performed (Stamatakis,

211 2014). Based on the inferred phylogenetic tree, 6,803 sequences regarded as

212 RHO orthologs were extracted for a scan of the amino acid residues at the sites 94 213 and 178.

214

215 Acknowledgments

216 We thank Rui Matsumoto and Kiyomi Murakumo at Okinawa Churaumi Aquarium

217 for their assistance in sampling. Our gratitude extends to Tomohiro Sugihara at

218 Osaka City University for his assistance in laboratory experiments. 


\section{Additional information}

\section{$221 \quad$ Funding}

222 This study was funded by grants awarded to $18 \mathrm{H} 02482$ and $21 \mathrm{H} 00435$ by MK.

\section{Author contributions}

226 S.K. and A.T. designed the study. K.Y., K.S. and M.K. performed research and 227 analyzed the data. S.K., K.Y., and M.K. drafted the manuscript, and all the authors 228 contributed to manuscript finalization.

229

\section{Author ORCIDs}

231 Kazuaki Yamaguchi 0000-0002-9890-7721

232 Mitsumasa Koyanagi 0000-0002-7741-918X

233 Akihisa Terakita 0000-0002-8379-8913

234 Shigehiro Kuraku 0000-0003-1464-8388

\section{References}

237 Alexander, N. S., Katayama, K., Sun, W., Salom, D., Gulati, S., Zhang, J., Mogi, 238 M., Palczewski, K., \& Jastrzebska, B. (2017). Complex binding pathways 239 determine the regeneration of mammalian green cone opsin with a locked 240 retinal analogue. Journal of Biological Chemistry, 292(26). https://doi.org/10.1074/jbc.M117.780478 
242

243

244

Al-Jandal, N., Farrar, G. J., Kiang, A. S., Humphries, M. M., Bannon, N., Findlay, J. B. C., Humphries, P., \& Kenna, P. F. (1999). A novel mutation within the rhodopsin gene (Thr-94-Ile) causing autosomal dominant congenital stationary night blindness. Human Mutation, 13(1). https://doi.org/10.1002/(SICI)10981004(1999)13:1

Capella-Gutiérrez, S., Silla-Martínez, J. M., \& Gabaldón, T. (2009). trimAl: A tool for automated alignment trimming in large-scale phylogenetic analyses. Bioinformatics, 25(15). https://doi.org/10.1093/bioinformatics/btp348

Elasmobranch Phylogeny: A Mitochondrial Estimate Based on 595 Species. (2012). In Biology of Sharks and Their Relatives. https://doi.org/10.1201/b11867-9

Fasick, J. I., Algrain, H., Serba, K. M., \& Robinson, P. R. (2019). The retinal pigments of the whale shark (Rhincodon typus) and their role in visual foraging ecology. Visual Neuroscience, 36. https://doi.org/10.1017/S0952523819000105

Hara, Y., Yamaguchi, K., Onimaru, K., Kadota, M., Koyanagi, M., Keeley, S. D., Tatsumi, K., Tanaka, K., Motone, F., Kageyama, Y., Nozu, R., Adachi, N., Nishimura, O., Nakagawa, R., Tanegashima, C., Kiyatake, I., Matsumoto, R., Murakumo, K., Nishida, K., ... Kuraku, S. (2018). Shark genomes provide insights into elasmobranch evolution and the origin of vertebrates. Nature Ecology and Evolution, 2(11). https://doi.org/10.1038/s41559-018-0673-5 
263

Hart, N. S., Lamb, T. D., Patel, H. R., Chuah, A., Natoli, R. C., Hudson, N. J., Cutmore, S. C., Davies, W. I. L., Collin, S. P., Hunt, D. M., \& Chang, B. (2020). Visual Opsin Diversity in Sharks and Rays. Molecular Biology and Evolution, 37(3). https://doi.org/10.1093/molbev/msz269

Janz, J. M., Fay, J. F., \& Farrens, D. L. (2003). Stability of dark state rhodopsin is mediated by a conserved ion pair in intradiscal loop E-2. Journal of Biological Chemistry, 278(19). https://doi.org/10.1074/jbc.M210567200

Katoh, K., \& Standley, D. M. (2013). MAFFT multiple sequence alignment software version 7: Improvements in performance and usability. Molecular Biology and Evolution, 30(4). https://doi.org/10.1093/molbev/mst010

Koyanagi, M., Wada, S., Kawano-Yamashita, E., Hara, Y., Kuraku, S., Kosaka, S., Kawakami, K., Tamotsu, S., Tsukamoto, H., Shichida, Y., \& Terakita, A. (2015). Diversification of non-visual photopigment parapinopsin in spectral sensitivity for diverse pineal functions. BMC Biology, 13(1). https://doi.org/10.1186/s12915-015-0174-9

Musilova, Z., Cortesi, F., Matschiner, M., Davies, W. I. L., Patel, J. S., Stieb, S. M., de Busserolles, F., Malmstrøm, M., Tørresen, O. K., Brown, C. J., Mountford, J. K., Hanel, R., Stenkamp, D. L., Jakobsen, K. S., Carleton, K. L., Jentoft, S., Marshall, J., \& Salzburger, W. (2019). Vision using multiple distinct rod opsins in deep-sea fishes. Science, 364(6440). https://doi.org/10.1126/science.aav4632 
284 Nakamura, I., Matsumoto, R., \& Sato, K. (2020). Body temperature stability in the

Palczewski, K., Kumasaka, T., Hori, T., Behnke, C. A., Motoshima, H., Fox, B. A., le Trong, I., Teller, D. C., Okada, T., Stenkamp, R. E., Yamamoto, M., \& Miyano, M. (2000). Crystal structure of rhodopsin: A G protein-coupled receptor. Science, 289(5480). https://doi.org/10.1126/science.289.5480.739

Park, P. S. H. (2014). Constitutively Active Rhodopsin and Retinal Disease. In Advances in Pharmacology (Vol. 70). https://doi.org/10.1016/B978-0-12-

Sato, K., Yamashita, T., Kojima, K., Sakai, K., Matsutani, Y., Yanagawa, M., 417197-8.00001-8 Yamano, Y., Wada, A., Iwabe, N., Ohuchi, H., \& Shichida, Y. (2018). Pinopsin evolved as the ancestral dim-light visual opsin in vertebrates. Communications Biology, 1(1). https://doi.org/10.1038/s42003-018-0164-x

Singhal, A., Guo, Y., Matkovic, M., Schertler, G., Deupi, X., Yan, E. C., \& Standfuss, J. (2016). Structural role of the T94I rhodopsin mutation in congenital stationary night blindness. EMBO Reports, 17(10). https://doi.org/10.15252/embr.201642671

302 Srinivasan, S., Fernández-Sampedro, M. A., Ramon, E., \& Garriga, P. (2017). 
Stamatakis, A. (2014). RAxML version 8: A tool for phylogenetic analysis and postanalysis of large phylogenies. Bioinformatics, 30(9). https://doi.org/10.1093/bioinformatics/btu033

Tyminski, J. P., de La Parra-Venegas, R., Cano, J. G., \& Hueter, R. E. (2015). Vertical movements and patterns in diving behavior of whale sharks as revealed by pop-up satellite tags in the Eastern Gulf of Mexico. PLoS ONE,

Yamaguchi, K., Koyanagi, M., \& Kuraku, S. (2021). Visual and nonvisual opsin genes of sharks and other nonosteichthyan vertebrates: Genomic exploration 10(11). https://doi.org/10.1371/journal.pone.0142156 K., Yamano, Y., Wada, A., Sako, Y., \& Shichida, Y. (2015). Origin of the low thermal isomerization rate of rhodopsin chromophore. Scientific Reports, 5. https://doi.org/10.1038/srep11081 


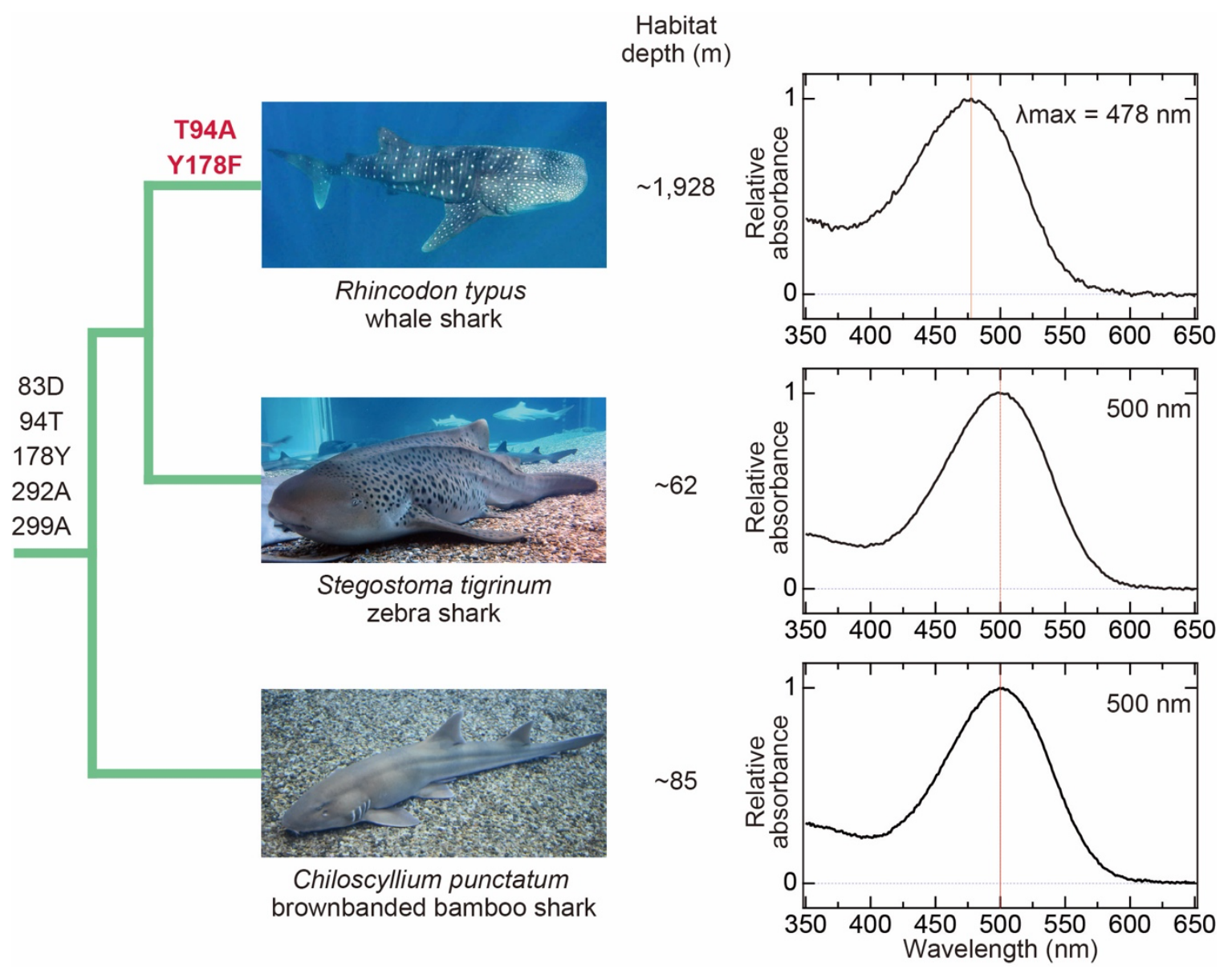

325 Figure 1. Absorption spectra of RHO pigments of the whale shark and its close

326 relatives. Their phylogenetic relationship is depicted with the amino acid residues

327 at novel (94 and 178) and previously documented (83, 292, and 299) spectral

328 tuning sites. Each absorption spectrum was normalized to the maximum

329 absorbance in the visible light region. The wavelengths of maximum absorbance of

330 the RHO pigment ( $\lambda \max )$ are based on this study (zebra shark) and existing

331 literature (Hara et al., 2018). 

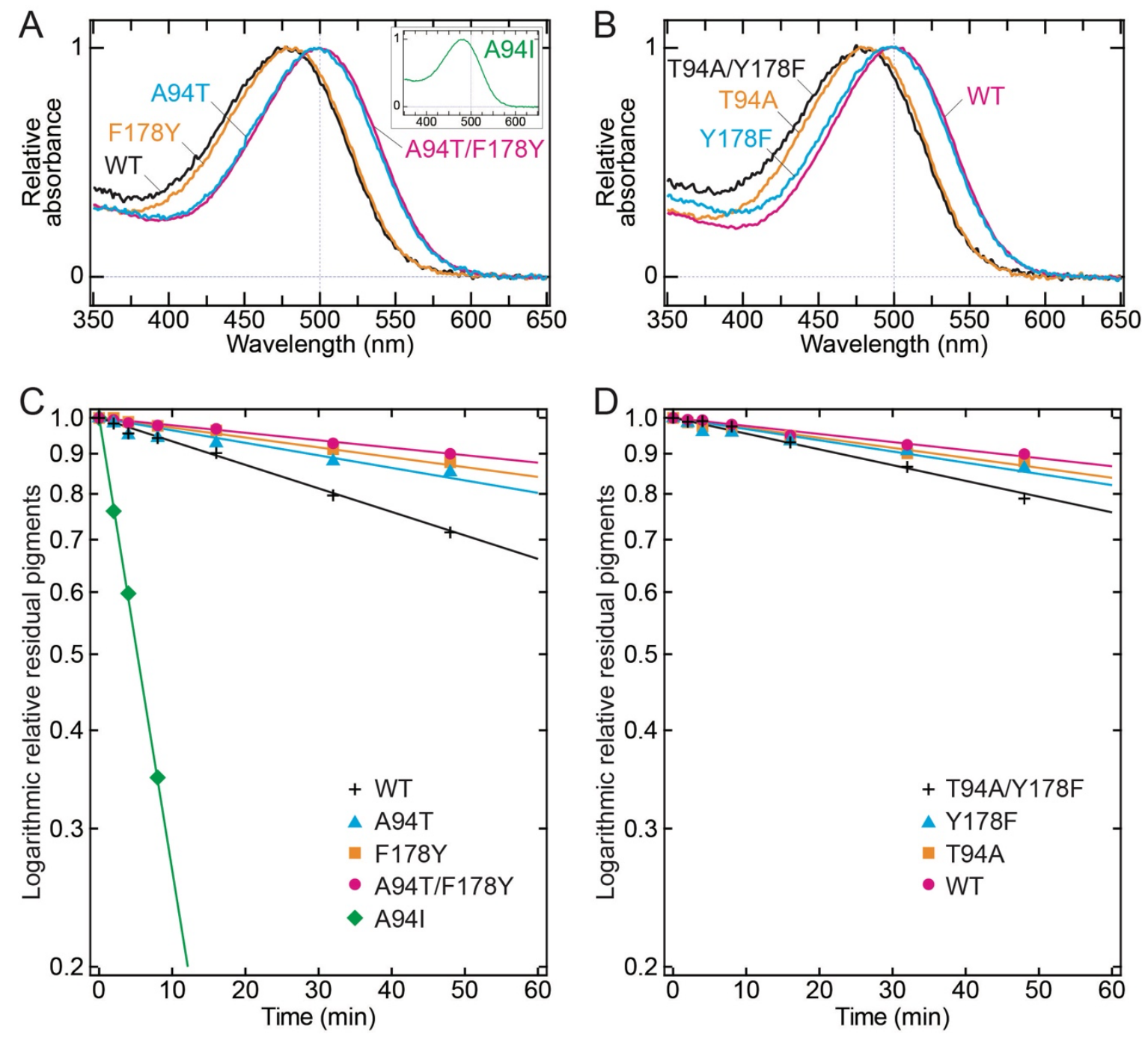

334 Figure 2. Experimental characterization of the whale shark and the zebra shark

335 RHOs. (A) Absorption spectra of the wild-type (WT, black), the A94T mutant (blue),

336 the F178Y mutant (orange), the A94T/F178Y mutant (magenta), and the A94I

337 mutant (green; inset) of the whale shark RHO. (B) Absorption spectrum of the wild-

338 type (WT; magenta), the T94A mutant (orange), the Y178F mutant (blue), and the

339 T94A/Y178A mutant (black) of the zebra shark RHO. (C) Time-course thermal

340 decay for the whale shark RHO. The colors correspond to those for the constructs

341 in A. The vertical axis shows relative amounts of residual RHO quantified with the 
bioRxiv preprint doi: https://doi.org/10.1101/2021.10.01.462724; this version posted October 1 2021. The copyright holder for this preprint

(which was not certified by peer review) is the author/funder, who has granted bioRxiv a license to display the preprint in perpetuity. It is made available under aCC-BY-NC 4.0 International license.

342 average absorbance of individual $\mathrm{RHO}$ samples at the 5 points $(\lambda \max -2, \lambda \max -1$,

$343 \lambda \max , \lambda \max +1$, and $\lambda \max +2 \mathrm{~nm})$, assuming that the absorbance immediately after

344 heating was 1 and the absorbance after light irradiation was 0. (D) Time-course

345 thermal decay for the zebra shark RHO. The colors correspond to those for the

346 constructs in $B$, and the vertical axis is shown in the same way as in $C$.

347

348 
349 Table 1. Spectral and thermal properties of the wild-type and mutant RHOs studied.

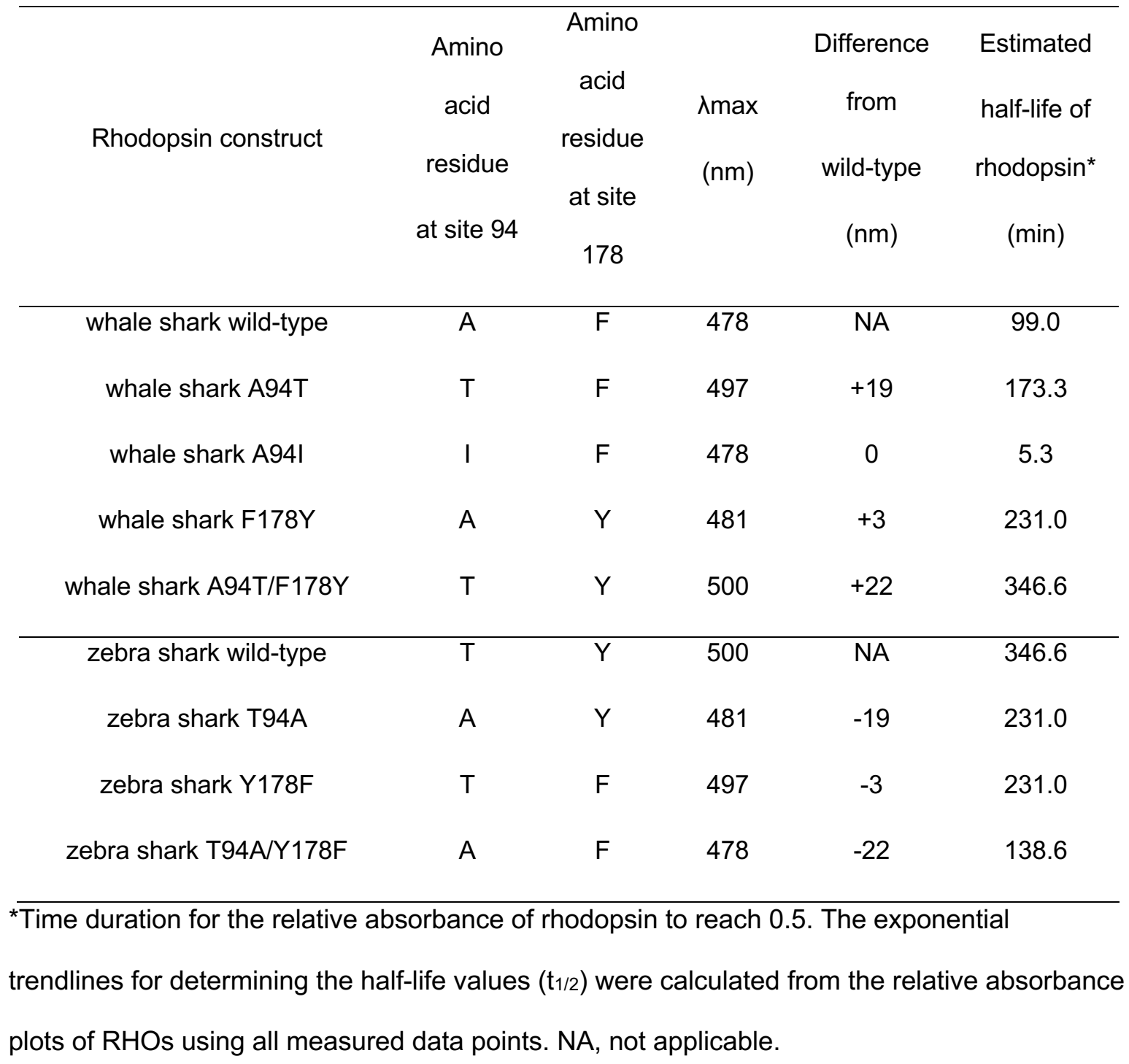


